\title{
Sprechakte unter besonderer Berücksichtigung der Höflichkeit
}

\author{
Tahir Balcı (D), Adana
}

https://dx.doi.org/10.37583/diyalog.958479

\begin{abstract}
Deutsch)
Die Sprache ist ein hervorragendes Werk, das durch Interaktion entsteht und mit dem wir eine Wirklichkeit erzeugen, die eine sowohl konstruierende als auch destruierende Rolle spielen kann. Manche Linguisten vergleichen den Sprachgebrauch mit dem Sprachspiel. Mit seiner Hervorhebung der Sprachverwendung und seinem einprägsamen und geschliffenen Ausspruch, Die Bedeutung eines Wortes ist sein Gebrauch in der Sprache' gilt Wittgenstein als Vorgänger der linguistischen Pragmatik. Seine Äußerungen inspirierten die Theoretiker der Sprechakttheorie.
\end{abstract}

Nach Austin verläuft der Sprachgebrauch in Sprechakten, wobei jeder Akt aus den Teilakten Lokution, Illokution und Perlokution entsteht und auch defizitäre Sprechakte möglich sind. Aus der Tatsache, dass der Anzahl der Kontexte keine Grenzlinie zu ziehen ist, dass es also unendlich viele Kontexte und dementsprechend so viele Funktionen gibt, können wir ebenfalls auf eine unendliche Zahl von expliziten oder impliziten Sprechakten schließen. Die Sprechakte haben einen modalen Charakter, der a) vom Blickpunkt des Sprechers und des Hörers, b) vom Kontext, in dem sich die Sprechakte abspielen, c) von der Gesellschaft als der Trägerin einer Kultur und d) von den Naturumständen, unter denen die Interaktanten leben, abhängt. Die Sprechakte gelingen nur dann, wenn die Interaktanten kooperationsbereit sind. Das allein genügt aber nicht; auch das Höflichkeitsprinzip ist sehr konstitutiv. In dieser Arbeit werden besondere türkische Sprechakte analysiert, die zeigen, dass die Äußerungstypen nicht immer mit den Illokutionen übereinstimmen. Das kann vom Kontext und auch von suprasegmentalen Merkmalen des jeweiligen Sprechaktes abhängen.

Schlüsselwörter: Pragmalinguistik, Interaktion, Sprechakte, Kooperationsbereitschaft, Höflichkeit.

Abstract (English)

\section{Speech Acts with Special Attention to Courtesy}

Language is an excellent work that arises through interaction and with which we create a reality that can play a constructive as well as a destructive role. Some linguists compare the use of language with the language play. With his emphasis on the use of language and his catchy and polished saying "The meaning of a word is its use in language", Wittgenstein is considered the predecessor of linguistic pragmatics. His statements inspired the most important theorist of speech act theory.. According to Austin, language use is in speech files, with each act arising from the sub-files location, illocation and perlocution, and deficient speech files are also possible. From the fact that there is no limit to the number of contexts, that is, there are infinitely many contexts and, accordingly, so many functions, we can also conclude from an infinite number of explicit or implicit speech acts. The speech acts have a modal 
character, which a) from the point of view of the speaker and the listener, b) from the context in which the speech acts take place, c) from society as the bearer of a culture and d) from the natural circumstances under which the interactants live, depend. The speech files are only successful if the interactants are willing to cooperate. But that alone is not enough; the principle of politeness is also very constitutive. In this work, special Turkish speech acts are analyzed, which show that the utterance types do not always correspond to the illocution. This can depend on the context and also on supra-segmental characteristics of the respective speech act.

Keywords: Pragmalinguistics, Interaction, Speech Acts, Willingness to Cooperate, Courtesy. 


\section{EXTENDED ABSTRACT}

Language is an excellent work that arises through interaction and with which we create a reality that can play a constructive as well as a destructive role. The consideration of language as a driving and constructive force can be traced back to L. Wittgenstein, who compared language use with language play. With his emphasis on the use of language and his catchy and polished saying "The meaning of a word is its use in language", he is considered the predecessor of linguistic pragmatics. His statement inspired the founder of "speech act theory"; J. L. Austin, who very often quoted Wittgenstein. According to Austin, language use can be analyzed in terms of speech acts; each utterance can be considered according to the related sub-category of the speech acts: locutionary, illocutionary, and perlocutionary acts; and also deficient speech acts.

It is assumed that there are over ten thousand types of speech acts, and besides depending on the context - other speech acts can be embedded in one speech act, says Alwin Fill. From the fact that there is no limit to the number of contexts and functions, an infinite number of explicit or implicit speech acts can be concluded from a single utterance.

The speech acts have a modal character, depending on a)the point of view of the speaker and the listener, b) the context in which the speech acts take place, c)society as the bearer of a culture and d)the natural circumstances under which the interactants are.

Studying language use, another approach was adopted by H.-J. Heringer. It was asserted under the title "Communication-constituting principles". The following principles can be applied to successful communication: Be relevant! Be informative! Be sincere! Speak clearly!

Certainly, there are debatable cases, considering the truth of an utterance. Determining the meaning of a speech unit is relational and depends on the communication partner's the point of view. For instance, imagine a student who cannot distinguish red from green. S/he says a fellow student: "Your eyes are red today, you have probably been cramming hard for the exam all night long", even though her eyes radiate a fresh green color. Many speech acts can follow this utterance, which can even offend the other person. Here, one can think of the color scale continuity, of the culture and necessity of the concept formation and of the prototype theory of Eleanor Rosch, which wants to classify the words according to their typical characteristics.

The same phenomenon can be expressed, perceived and assessed differently by different people. This has nothing to do with the language's inability to express something. The different perception and evaluation are not something that is linguistically enforced. Whether or not a language has a form for a content is primarily related to whether the users of that language need this form or not. The content is a reality, but it can be integrated into various forms. Guy Deutscher makes this obvious using the example of the coordinate system, which can be geographical (south, north, west, east) or egocentric (front, back, left, right). His remarks show that there are languages with unusual ways of speaking about spatial relationships, whose modes of 
expression differ from those previously thought to be universal and natural. This means that spatial thinking and speaking presupposes a cultural convention that is shaped by non-linguistic nature. The way in which we deal with nature and the environment influences our perception of language and the implementation of speech acts. Therefore, different words can associate very different things with different people. We must, therefore, always bear in mind that a speech act maynot have the intended effect. All these concerns indicate us that the implications of speech acts are clearly modal.

The speech files are only successful if the interactants are willing to cooperate. However, this alone is not enough; the principle of politeness is also very constitutive. In this work, special Turkish speech acts are analyzed, which show that the utterance types do not always correspond to the illocution. This can depend on the context and also on supra-segmental characteristics of the respective speech act. 


\section{Einleitung}

Die Sprache ist ein hervorragendes Auseinandersetzungswerk des Menschen, das durch Interaktion mit der Umwelt oder mit sich selbst entsteht und mit dem wir im Watzlawick'schen Sinne eine Wirklichkeit erzeugen, die eine sowohl konstruierende als auch destruierende Rolle spielen kann. Schon hunderte Jahre vor Chr. hatten die griechischen Philosophen Sokrates und Platon diese Rolle der Sprache erkannt. Sokrates diskutierte am Markplatz gern mit jungen Leuten über erbauende philosophische Themen wie Sein, Wahrheit, Erkenntnis, Schönheitsliebe und menschliche Tugenden. Und Platon wies in seinem berühmten Dialog Kratylos auf die Funktion der Sprache als Manipulations- und Täuschungsmittel hin, dass die Sophisten zu seiner Zeit angewandt haben sollten (s. Fill 2013: 15-17, 139; Aksöz/ Balc1 2019; Aksöz 2017).

Die Betrachtung der Sprache als treibende und konstruierende Kraft geht vor allem auf L. Wittgenstein zurück, der den Sprachgebrauch mit dem Sprachspiel verglichen hatte. Mit seiner Hervorhebung der Sprachverwendung und seinem einprägsamen und geschliffenen Ausspruch „Die Bedeutung eines Wortes ist sein Gebrauch in der Sprache“ gilt er als Vorgänger der linguistischen Pragmatik (vgl. Schroeder 2015: 1). Diese und ähnliche sentenzartige Äußerungen inspirierten viele Wissenschaftler, so z. B. den britischen Philosophen \& Linguisten John Langshaw Austin.

\section{Sprechakte}

Mit seinem Werk How to Do Things with Words / Wie tut man Dinge mit Worten? begründete J. L. Austin die sog. Sprechakttheorie, nach der eine Äußerung die folgenden Teilakte aufweist:

Lokution bzw. lokutionärer Akt: Ich artikuliere ein komplexes Schallgebilde, das zu wohlgeformten Wörtern meiner Sprache wird und somit eine Bedeutung/Funktion hat bzw. mit dem ich mich auf Gegenstände, Vorgänge, Tätigkeiten oder Zustände beziehen kann.

Illokution bzw. illokutionärer Akt: Mit meiner Äußerung tue oder beabsichtige ich etwas. Wenn ich also aus Lauten meiner Sprache sinnvolle Wörter bilde, vollziehe ich eine bestimmte Handlung, von der ich erwarte, dass sie beim Hörer zur Wirkung kommen soll.

Perlokution bzw. perlokutionärer Akt: Aus meinem intentionellen Tun ergeben sich für den Hörer Konsequenzen. Er entgegnet entweder durch einen Sprechakt oder geht meinen Erwartungen / Verpflichtungen nach (vgl. Maas/ Wunderlich 1972: 119; Fill 2013: 201; Balc1 2017: 334).

Maas \& Wunderlich (1972: 121f.) sind aber der Meinung, dass es linguistisch unvollständige Sprechakte gibt, in denen nicht alle Teilakte realisiert zu werden brauchen. Wenn man z. B. etwas diktiert, etwas Diktiertes vorliest bzw. etwas Gesagtes wiederholt, werde im Grunde keine gegenseitige Beziehung intendiert. 
Wir sind hier anderer Ansicht. Wenn wir den Studierenden etwas diktieren, sagen wir ihnen damit einen Stoff zur Aufnahme bzw. zur Niederschrift an. Das wäre ein Befehl, und wenn sie das Gesprochene mitschreiben, ist der Sprechakt vollzogen. Wer nicht mitschreibt, hat den Sprechakt auch vollzogen. Er meldet damit nur einen Einspruch / Protest / Unwillen / Bruch der Verpflichtung an und sagt: Ich tue das Gegenteil davon, was sie uns erteilen.

Ebenda finden sich sehr interessante Sprechakt-Beispiele. Es gebe illokutive Akte ohne Folgen für die Kommunikationssituation; wenn z. B. ein Lehrling in die Firma kommt und seinem Meister „Guten Morgen, Herr Werner“ sagt, brauche der Meister nicht zu erwidern. Bei der Nicht-Ausführung dieses Aktes in derselben Kommunikationssituation müsse der Lehrling aber mit Konsequenzen rechnen, die einen soziokulturellen Hintergrund haben.

Dass der Meister nicht mit einem „Guten Morgen“ zu erwidern braucht, heißt m. E. nicht, dass der illokutive Akt des Lehrlings keinen Effekt und keine Folgen beim Meister gefunden hat. Eine der Vorbedingungen für das Stattfinden der Kommunikation ist die Kooperation und die Korrektheit. Im Normalfall ist $\mathrm{zu}$ erwarten, dass der Lehrling den Meister grüßt. Falls er ihn nicht grüßt, ist wiederum eine andere Intention (eine andere Illokution) mit dem Nicht-Grüßen zu verbinden. Aus irgendeinem Grund kann der Lehrling ärgerlich / missgelaunt sein und vom Meister eine Unterstützung erwartet haben. Das kann man als eine Bitte verstehen. Und wenn der Lehrling den Meister grüßt und der Gruß ohne Reaktion bleibt, ist das einerseits keine kooperative Kommunikationssituation, weil jeder normale Meister antworten würde. Wenn der Meister nicht antworten würde, ist andererseits damit der illokutive Akt erfüllt, zumal der Meister den Gruß wahrgenommen hat und sich das Recht beimisst, nicht zu antworten. Das Schweigen / die Reaktionslosigkeit des Meisters ist also eine Art der Perlokution.

John Searle klassifiziert die Illokutionen in fünf Gruppen (vgl. Meibauer 2001: 95):

Mit Repräsentativa/ Assertiva verpflichtet sich der Sprecher auf die Wahrheit des Ausgedrückten (vermuten, sagen, behaupten, mitteilen, berichten, schwören): Ich nehme an, dass du selbst diese Arbeit geschrieben hast.

Mit Direktiva versucht der Sprecher, das Gegenüber zu einer Handlung zu bewegen oder sie zu unterlassen (fragen, bitten, raten befehlen, vorschlagen, nahelegen): Du könntest kommen, oder?

Durch Kommissiva entsteht eine Verpflichtung (versprechen, drohen, vereinbaren, garantieren, anbieten): Wir werden sie in der Kaufhalle treffen.

Expressiva dienen dazu, die eigene Gefühlslage auszudrücken (schimpfen, danken, entschuldigen, begrüßen, gratulieren): Die Unfairness in seiner Bildungskarriere ist beunruhigend.

Durch Deklarativa wird das Gegenüber institutionell (amtlich durch Schule, Kirche, Firma, Familie) für etwas verantwortlich gemacht bzw. mit etwas beauftragt 
oder ausgestattet, sodass es entsprechend dem Gesagten etwas vollzieht oder durch das Gesagte etwas vollzogen ist. Das Gesagte stimmt mit der Realität überein (ernennen, entlassen, beurkunden, verurteilen taufen): Ich verschreibe dir Aspirin.

Nach J. L. Austin gibt es über 10.000 Arten von Sprechakten und gleichzeitig können in einem Sprechakt - je nach dem Kontext - weitere Sprechakte eingebettet sein (vgl. Fill 2013: 200f.). Aus der Tatsache, dass der Anzahl der Kontexte keine Grenzlinie $\mathrm{zu}$ ziehen ist, dass es also unendlich viele Kontexte und dementsprechend so viele Funktionen gibt, können wir ebenfalls auf eine unendliche Zahl von expliziten oder impliziten Sprechakten schließen.

Nach Fill (2013: 203) hängen die Sprechakte mit dem Dialog zusammen. Eine ähnliche Betrachtungsweise war schon vor Fill bei Heringer u. a. (1977: 173) unter dem Titel „Kommunikationskonstituierende Prinzipien“ zu sehen:

- Sei relevant!

- Sei informativ!

- Sei aufrichtig!

- Sprich klar!

Zur Erläuterung des Aufbaus der Kommunikation und ihrer Prinzipien geben Heringer u. a. (1977: 167f.) den folgenden jiddischen Witz, der auf einer voreingenommenen Lüge basiert, als Beispiel

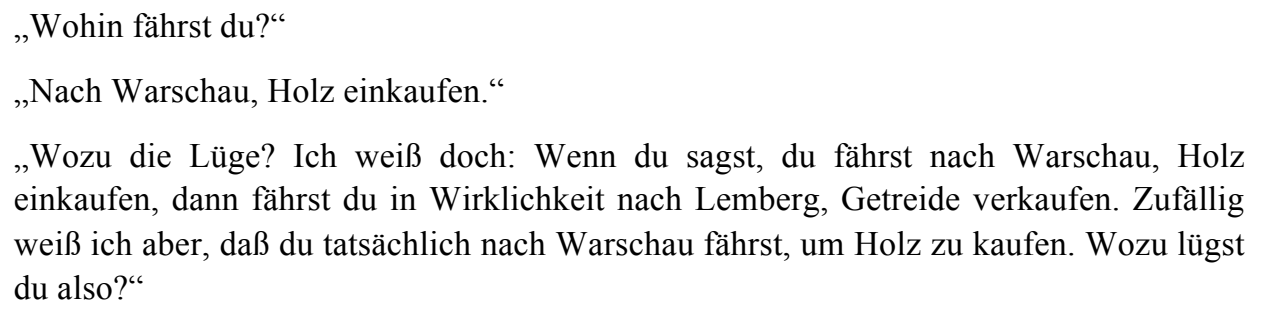

und zeigen, dass hier „die Wahrheit gewissermaßen zur Lüge zweiten Grades“ gemacht wird. Zur Vermeidung solcher Kommunikationshürden setzen sie das gegenseitige Vertrauen der Kommunikationsteilnehmer voraus:

Misstrauen belastet sie. Prinzipielles Misstrauen vollends führt zum Zusammenbruch jeder Kommunikation... Wir können uns sagen, daß dies doch nur ein Witz ist... So etwas kommt im Leben vor und ist in gewissen Bereichen sogar die Regel. (ebd.)

Das alles deutet darauf hin, dass die Sprechakte nicht über die Striktheit mathematischer Formeln oder gerichtlicher Urteile verfügen und von vielen Umständen abhängen.

\section{Der modale Charakter der Sprechakte}

Ein wichtiger Wesenszug der Sprechakte ist ihre Modalität. Die Art und Weise, wie Sprechakte geschehen oder ausgeführt werden, d. h. die Bedingungen, Verhältnisse und Umstände, mit denen sie verbunden sind, sind sehr oft relational, kulturbezogen und naturgebunden. 


\section{Die Wahrheit der Sprechakte kann relational sein}

Sicherlich gibt es diskutable Zweifelsfälle, in denen die Wahrheit einer Äußerung relational ist und vom Blickpunkt eines Kommunikationspartners abhängt. Stellen wir uns einen Studierenden vor, der zwischen Rot und Grün nicht unterscheiden kann und einer Kommilitonin sagt, „Deine Augen sind heute rot, du hast wohl die ganze Nacht mächtig gepaukt", obwohl ihre Augen ein frisches Grün ausstrahlen. Auf diese Äußerung können viele Sprechakte folgen, die das Gegenüber auch beleidigen können. Man erinnere sich hier an die Kontinuität der Farbenskala, an die Kultur- und Bedarfsbedingtheit der Begriffsbildung und an die Prototypentheorie von Eleanor Rosch, die die Wörter nach für sie typischen Merkmalen einstufen will. Bis zu welcher Größe ist z. B. ein Wurm klein?

\section{Die Wahrheit der Sprechakte kann kulturbezogen und naturgebunden sein}

Dasselbe Phänomen kann von verschiedenen Menschen anders ausgedrückt, wahrgenommen und beurteilt werden. Das hat nicht mit der Unfähigkeit der jeweiligen Sprache zu tun, etwas auszudrücken. Auch die unterschiedliche Wahrnehmung und Wertung ist nicht etwas sprachlich Erzwungenes. Ob eine Sprache für einen Inhalt eine Form hat oder nicht, hat in erster Linie damit zu tun, ob die Benutzer dieser Sprache dieser Form bedürfen oder nicht. Der Inhalt ist eine Realität, wird aber in eine andere Form integriert. Guy Deutscher macht das am Beispiel des Koordinatensystems offensichtlich, das geographisch (Süden, Norden, Westen, Osten) oder egozentrisch (vorn, hinten, links, rechts) sein kann. Seine Ausführungen zeigen, dass es Sprachen mit ungewöhnlichen Arten des Redens über räumliche Verhältnisse gibt, deren Ausdruckweisen von den bis dahin für universell und natürlich gehaltenen abweichen. Er spricht von exotischen Sprachen, in denen die Hauptachse der Richtungen durch die oppositionellen landeinwärts vs. seewärts (deine Seewärts-Wange!) oder durch Geländemerkmale (Ist das warme Wasser in dem Bergauf-Hahn?) ausgedrückt wird. Der Richtungssinn sei etwas Selbstverständliches und unerklärbar, genauso woher wir wüssten, wo vorne/hinten/links/rechts sei. Es gebe auch andere Anhaltspunkte als die oben erwähnten, die bei der Fixierung der Begriffsbildung, beim Orientierungsgefühl und beim Sprachgebrauch mitspielen, z. B. die Helligkeitsunterschiede an den Seiten der Stämme bestimmter Bäume, die Ausrichtung von Termitenhügeln / Windrichtungen, der Flug von Fledermäusen und Zugvögeln, die Lage von Sanddünen im Küstengebiet (vgl. Deutscher 2014: 186-199). Das bedeutet, dass räumliches Denken und Sprechen eine kulturelle Konvention voraussetzt, die von der nichtsprachlichen Natur gestaltet wird. Die Art und Weise, wie wir mit der Natur und mit der Umwelt umgehen, beeinflusst unsere Sprachvorstellung und die Realisierung der Sprechakte, sodass verschiedene Wörter bei verschiedenen Leuten ganz Verschiedenes assoziieren können. Daher müssen wir immer damit rechnen, dass ein Sprechakt nicht die intendierte Wirkung haben kann (vgl. hierzu auch Zengin 2004).

Das alles zeigt uns, dass die Implikationen der Sprechakte einen eindeutig modalen Charakter haben, der 
- vom Blickpunkt des Sprechers und des Hörers,

- vom Kontext, in dem sich die Sprechakte abspielen,

- von der Gesellschaft als der Trägerin einer Kultur,

- von den Naturumständen, unter denen die Interaktanten leben, abhängt.

\section{Die Sicherung der Kooperation}

Die Kommunikation bzw. die Sprechakte gelingen nur dann, wenn die Interaktanten kooperationsbereit sind. Dabei gibt es eine bestimmte Situation und Kommunikationspartner, die ein gemeinsames Ziel haben und von denen ,jeder“ bestimmte Teilaufgaben übernehmen muss. Die Verletzung dieses Prinzips führt zum Misslingen des Dialogs und zur Täuschung des Partners. Zur Sicherung der Kooperation und zur Überwindung möglicher Kommunikationsprobleme müssen die Kommunikationsteilnehmer die Verantwortung für die kommunikationskonstituierenden Prinzipien Relevanz + Informativität + Aufrichtigkeit + Klarheit tragen. Nach Fill (2013: 226) kann man dadurch zum Gelingen der Kommunikation und zur intermenschlichen Bandstiftung beitragen, indem man jeglicher Abwertung, jeglichem Streit und Konflikt ausweicht und folgende Sprechweisen vermeidet:

\section{Höflichkeit - Unhöflichkeit}

Das Kooperationsprinzip allein genügt für das Gelingen der Kommunikation nicht; auch das Höflichkeitsprinzip, das oben von A. Fill durch die abgeratenen Sprechweisen indirekt suggeriert wird, damit einem das sprachliche Handeln glückt, ist sehr konstitutiv. Die abgeratenen Sprechweisen weisen genau auf die Vermeidung der Verletzung der Persönlichkeit des kommunikativen Gegenübers, was Höflichkeit genannt wird:

\footnotetext{
Höflichkeit kann jedoch sowohl eine direkte Hinwendung zum Gegenüber sein, in der sich der Sprecher/die Sprecherin mit dem Gegenüber direkt solidarisch zeigt, als auch kommunikativ indirektes Handeln, das dem Gegenüber mehr Optionen lässt, sich zustimmend oder ablehnend $\mathrm{zu}$ den Kommunikationshandlungen seines Gesprächspartners zu verhalten. [Man] bezeichnet positive und negative Höflichkeit deshalb auch als Solidaritäts- und Distanzhöflichkeit.“ (Kuße 2012: 86)
}

Nach G. Leech lässt sich die Höflichkeit als Strategie zur skalaren Verstärkung oder Abtönung der illokutionären Sprechakte einsetzen, die in Anlehnung an S. Bonacchi (2013: 58f.) als Maximen und Submaximen wie folgt zusammengefasst werden können:

1) Die Takt-Maxime: 1a) Minimiere den Aufwand/die Kosten für den Anderen! Darf ich Dich einen Augenblick sprechen? 1b) Maximiere den Nutzen für den Anderen! Du kannst gern den Kuchen aufessen!

2) Die Maxime der Großzügigkeit: 2a) Minimiere den Nutzen für dich selbst! Mir macht es nichts aus, ich sage es für dich. 2b) Maximiere den Aufwand/die Kosten 
für dich selbst! Komm einfach bei uns vorbei, ich bereite etwas vor und wir können die Sache beim Abendessen in aller Ruhe besprechen!

3) Die Maxime der Zustimmung: 3a) Minimiere die Kritik/Geringschätzung des Anderen! Es war bestimmt sehr lustig gestern bei der Fete! (statt: „es war zu laut!“) 3b) Maximiere die Wertschätzung des Anderen! Wenn du mir helfen könntest, wäre es super, du bist ein Genie in Computerdingen!

4) Die Maxime der Bescheidenheit: 4a) Minimiere die Wertschätzung deines Selbst! (Üblich in den Antworten auf Lob und Komplimente). 4b) Maximiere die Geringschätzung deines Selbst! Wie dumm von mir! (statt: *Wie überaus klug von mir!)

5) Die Maxime der Übereinstimmung: 5a) Minimiere die Unstimmigkeiten zwischen dir und dem Anderen! 5b) Maximiere Übereinstimmungen zwischen dir und Anderen!

6) Die Maxime der Sympathie: 6a) Minimiere die Antipathie zwischen dir und dem Anderen! 6b) Maximiere die Sympathie zwischen dir und dem Anderen! (vgl. Bonacchi 2013: 58f.; vgl. auch Steinbach 2007: 244). Wenn man von Höflichkeit spricht, so fällt einem direkt der Begriff Unhöflichkeit ein, welche oben im Kontext der $\mathrm{zu}$ vermeidenden Sprechweisen angedeutet wurde. In den Wörterbüchern wird die Höflichkeit mit folgenden Eigenschaften erklärt: wohlerzogen, verbindlich, taktvoll, rücksichtsvoll, zuvorkommend, liebenswürdig, gefällig, hilfsbereit, entgegenkommend, freundlich, verbindlich, galant, verständnisvoll, wohlwollend, gefällig, angenehm, ansprechend, einladend, gutgesinnt, gütig [...]. In der wissenschaftlichen Literatur werden die Begriffe nicht-höflich und unhöflich nicht synonymisch gebraucht, wobei hauptsächlich die folgenden Adjektive mit unhöflich verbunden werden: brüsk, ungeschliffen, taktlos, ungefällig, ungehobelt, unwirsch, gebieterisch, verneinend, grob, schroff, rau, rüde, unfreundlich, abweisend. Unhöflichkeit und Höflichkeit unterliegen verschiedenen Gebrauchsbedingungen. Während die Höflichkeit mit bandstiftenden Charaktereigenschaften wie Takt, Großzügigkeit, Bescheidenheit, Wertschätzung des Gegenübers, Sympathie, Toleranz und Kompromissbereitschaft in Zusammenhang gebracht wird und immer als erwünscht gilt, ist die Unhöflichkeit verpönt, sogar manchmal tabuisiert, und der Tabu-Bruch führt nicht selten zu Streitigkeiten oder zum Bruch der Interaktion zwischen den Menschen, weil sie (die Unhöflichkeit) einen antidialogischen Charakter hat und eine dominante Machtausübung darstellt. Bonacchi (2013: 157f.) spricht in Anlehnung an Austin, Beebe und Culpeper von Unhöflichkeitsakten, die folgendermaßen klassifiziert werden:

1) Der Arrogativ, mit dem man die eigene Überlegenheit zeigen will.

2) Der Offensiv (der Aggressiv), wodurch Beleidigung bzw. Angriff gegen den Anderen stattfindet.

3) Mit dem Limitativ zielt man darauf ab, durch die Kontrolle des Anderen bzw. durch die Einschränkung seines Handlungsspielraums Macht auszuüben. 
4) Der Unreziproke ist antidialogisch und verletzt die Gegenseitigkeit und die Anerkennung des Anderen.

\section{Besondere Sprechakte aus dem türkischen Sprachgebrauch}

Besonders unter Männern und den meisten Jugendlichen jeden Geschlechts sind Vulgarismus und Sarkasmus Kennzeichen des alltäglichen türkischen Sprachgebrauchs. In einem Bekanntenkreis oder bei einem geselligen Beisammensein können ganz neutrale Begriffe absichtlich verdreht und mit nicht dazugehörigen semantischen Merkmalen belastet werden. Hierbei geht es in den meisten Fällen um Wörter bzw. Begriffe, die metaphorisch auf den Sexualbereich bezogen werden und damit schlüpfrige unanständige Obszönitäten hervorrufen, die das Schamgefühl verletzen. Um nur ein ganz einfaches Beispiel zu geben, stelle man sich das Verb „vermek“ (dt. ,geben') vor, besonders wenn es ohne Objekt gebraucht wird. Uns geht es hier aber nicht um Sprechakte als obszönen Sprachgebrauch durch Metaphorik, sondern um besondere Sprechakte, die als Perlokution entweder nur Schweigen, Billigung und Missbilligung erlauben / erfordern.

In den letzten Jahren seines Lebens hat der Verfasser (T. B.) von seinem verstorbenen Vater sehr oft die folgenden Äußerungen gehört, die wohl auch dem Leser dieser Arbeit aus seinem Bekanntenkreis nicht unbekannt sein dürfen:

(1) Akranlarım bir bir öldü, galiba sira bize geldi (Gleichaltrige sind nacheinander gestorben, ich denke, es ist Zeit für mich).

(2) Ömrümüzün sonuna geldik (Ich bin am Ende des Lebens).

(3) Artık bir ayağımız mezarda (Ich stehe schon mit einem Fuß im Grab).

(4) Ömrümüzü doldurduk artık (Ich bin fast am Ende des Lebens).

(5) Yaşayacağımızı yaşadık (Ich habe schon genug gelebt /Ich habe alles erlebt, was erlebt werden kann).

Diese fast gleichbedeutenden Äußerungen drücken die gleiche Illokution aus, die jeder verhältnismäßig alte Mensch aufgrund seines Alters und/oder seines ungesunden Wesens fühlen würde. Anscheinend stellte der Vater fest, dass er sich dem Tode sehr genähert hat. Obwohl diese Äußerungen in Wirklichkeit / denotativ als Feststellung gelten können, sind sie gar nicht als solche intendiert. Mit diesem angeblich assertiven Sprechakt, der den Sprecher zur Wahrheit der ausgedrückten Proposition (Feststellung, Beschreibung, Behauptung, Explikation, Diagnose) verpflichten sollte, will der Vater keine Feststellung der Todes-Nähe machen; er äußert seinen Lebenswillen und seine Angst vor dem Tod, die er als einen inneren Konflikt erlebt und überwinden will. Schweigen gegenüber solchen Illokutionen ist nicht die erwartete, aber trotzdem annehmbare Reaktion. Auch nonverbale Signale wie Nicken, Lachen und besorgtes Gesicht wären nicht weniger unhöflich und verpönt als die Billigung der scherzhaft, ironisch oder auch bescheiden gemeinten Illokutionen, mit denen er eigentlich genau das Gegenteil intendiert. Der Sprecher erwartet eine konduktive Äußerung, will also, 
dass das Gegenüber sich auf ihn einstellt und ihn erleichtert, indem er das Geäußerte etwa wie folgt missbilligt:

(6) Hayır baba, Allah'a şükür sağlığın yerinde. Allah uzun ömürler versin! Kim kimden önce ölür bilinmez! (Nein, Papa, Gott sei Dank bist du in guter Gesundheit. Möge Allah dir ein langes Leben geben! Wer weiß, wer vor wem schon stirbt!).

In Anlehnung an A. Pande kann man solche Sprechakte samt ihren Teilakten mit der Einteilung der Kulturen als High Context und Low Context von Hall/ Hall ${ }^{1}$ begründen,

[...] nach der in den High Context Kulturen die Menschen enge Beziehungen zu ihren Mitmenschen haben, ... Linguistisch betrachtet brauchen diese Menschen indirekte Ausdrücke der Sprechakte, die vieles Unausgesprochene der Interpretation der Empfänger überlassen. In Indien würde man selten eine direkt vorgetragene Bitte oder eine direkt ausgesprochene Entschuldigung hören. (Pande 2009: 344)

Ähnlich sieht es auch aus bei den folgenden Äußerungstypen, mit denen der Sprecher sich abwertet, indem er sich selbst ironisiert, beschimpft oder beschuldigt: ${ }^{2}$

(7) Ben ne biçim insanım ya! (Was für ein Mensch bin ich!)

(8) Ben aptalım ya! (Ich bin ja dumm!).

(9) Ben ne geri zekalıım be! (Was für ein verdammter Idiot bin ich!).

Obwohl der Sprecher wörtlich behauptet, ein Dümmling oder ein Idiot zu sein, ist diese Selbstkritik nicht ernst zu nehmen. Der Sprecher kann wohl etwas gemacht haben, wegen dessen er sich kritisiert und daher entschuldigen will. Dafür verstellt er sich durch die Selbstironie bzw. Selbstbeschuldigung in eine andere Sphäre, baut sich somit eine Bewertungskluft zwischen dem Geäußerten und Gedachten, um sich durch Missbilligung des Geäußerten (Etwa: Nein, du bist gar nicht dumm! Jeder würde dasselbe tun!) vom Kommunikationspartner zu beschwichtigen. Die Billigung des Geäußerten (Und wie! Du bist wirklich dumm!) wäre ein nicht intendierter verpönter Unhöflichkeitsakt, der das Abgewertete abwertet. Selbst eine Billigung mit schwächerem Ton (Du müsstest doch vorsichtiger sein!) gilt als unhöflich.

(10) Senin işin iki saatimi aldı. - Benim için değmez mi? (Deine Sache hat mich zwei Stunden gekostet. - Lohnt es sich nicht für mich?).

Im obigen Dialog beschwert sich ein Dozent bei seiner Kollegin über die Mühe, die er sich für ihre Sache gegeben hat. Sie reagiert mit der Frage, ob es sich für sie nicht lohnt. Ist es möglich bzw. angemessen, dass er die Frage denotativ versteht und Değmez! sagt? Nein! Das wäre wiederum ein Verstoß gegen die Maxime der Großzügigkeit (Minimiere den Nutzen bzw. maximiere den Aufwand/die Kosten für dich selbst!), aber auch eine

\footnotetext{
${ }^{1}$ „High Context - Man nimmt an, dass die anderen den Zusammenhang genau kennen. - Nachrichten und Mitteilungen enthalten Kernpunkte. - Einzelheiten werden nicht erwartet, da jeder durch ein informelles Informationsnetz detailliert informiert ist. Low Context - Man muss ständig nachdenken, ob der Informationsstand der anderen auf dem richtigen Niveau ist. - Jede zweckdienliche Information wird nachdrücklich mitgeteilt. - Eine Fülle von Informationen von detaillierten Einzelheiten wird in jedem Fall zu jeder Entscheidung erwartet.“(Hall, E.T./ Hall M.R.; zit. n. Pande 2009: 339f.).

${ }^{2}$ Vgl. Für die Verwendung rhetorischer Mittel in verschiedenen Äußerungsformen Aksöz (2017).
} 
Verletzung der Bescheidenheit (Minimiere die Wertschätzung bzw. maximiere die Geringschätzung deines Selbst!).

Schimpfworte und Flüche gehören zum alltäglichen Sprachgebrauch, weil es in unserem Gesellschaftsleben viele gesellige oder streitige Kontexte gibt, die dies erfordern. So ein vulgärer Sprachgebrauch entspricht aber nicht jedem Sprachgeschmack und hängt stark vom Erziehungsgrad der Sprachbenutzer ab. Trotzdem kann man behaupten, dass Äußerungen/ Schimpfworte wie die folgenden zum ungezwungenen vertraulichen familiären Sprachgebrauch gehören: ${ }^{3}$

(11)Hayvan! (Tier!).

(12)Eşek oğlu eşek! (Eselssohn!).

Wie sind nun diese Schimpfworte zu verstehen, die den Zorn des Sprechers ausdrücken? Wir erinnern uns an Situationen, in denen die Kinder so angesprochen wurden und so antworteten: Vergiss aber nicht, dass du meine Mutter / mein Vater bist! So eine Antwort ist offensichtlich gegenüber den Eltern unhöflich, genauso wie die Äußerung der Eltern. Beide Akte tragen nicht zur Lösung von Konflikten bei und eröffnen neue Sprechakte, die in gegenseitige Verletzung des Gegenübers münden und den Konflikt schärfen.

\section{Fazit}

Wenn eine Sprache da ist, ist sie für die Menschen da, die sie unter bestimmten Konventionen anwenden. Die Menschen benutzen die Sprache für die Auseinandersetzung mit der sozialen Umwelt, aber auch mit sich selbst. Dadurch überwältigen sie die Umwelt, lösen sie ihre Probleme oder rufen sie neue Konflikte hervor.

Das alles läuft in Sprechakten ab, die eine deutliche Modalität (vgl. auch Zengin 2003: 33-54) aufweisen, deren Implikationen a) vom Blickpunkt des Sprechers und des Hörers, b) vom Kontext, in dem sich die Sprechakte abspielen, c) von der Gesellschaft als der Trägerin einer Kultur und d) von den Naturumständen, unter denen die Interaktanten leben, abhängen.

Die Äußerungstypen stimmen nicht immer mit den Illokutionen überein. Das Geäußerte kann öfters das Gegenteil des Gedachten meinen. Das alles hängt vom Kontext und auch von suprasegmentalen Merkmalen des jeweiligen Sprechaktes ab.

Das Gelingen einer Illokution unterliegt der Einhaltung bestimmter Kommunikationsprinzipien und der Vermeidung von Sprechweisen und Handlungstypen wie Arrogativ/ Offensiv/ Limitativ/ Unreziproke, die die Höflichkeit verletzen und Konflikte stiften. Takt, Großzügigkeit, Wertschätzung des Anderen, Bescheidenheit, Sympathie und Kooperationsbereitschaft sind kommunikative

\footnotetext{
${ }^{3}$ Wir haben sogar schwerere, auf den Sexual- und Fäkalbereich bezogene und das Schamgefühl verletzende obszöne Schimpfworte unter Schüler*innen gehört, die sie beim Spaßmachen benutzten und die wir hier nicht schreiben können.
} 
Tugenden, die die interaktionale Höflichkeit garantieren. So haben wir Sprechakte festgestellt, mit denen der Sprecher von seinem Kommunikationspartner eine ganz bestimmte Perlokution erwartet, deren Verletzung zu Unhöflichkeitsakten führt.

\section{Literaturverzeichnis}

Aksöz, Munise (2017): Biçemler ve Uygulamalı Öğretimi. Konya: Çizgi Kitabevi Yayınları.

Aksöz, Munise / Balcı, Umut (2019): Die Sprache auf dem Basar, Balcı, Tahir, Öztürk, Ali Osman/ Serindağ, Ergün (Hg.): Schriften zur Sprache und Literatur III (43-50), London: Ijopec.

Balcı, Umut (2017). Kursbuch zur Prüfung ÖABT, Ijopec Publication, London.

Bonacchi, Silvia (2013): (Un)Höflichkeit Eine kulturologische Analyse Deutsch-Italienisch-Polnisch, Frankfurt a. M. et al, P. Lang. | verfügbar unter: https://www.academia.edu/7086781/_Un_H\%C3\%B6flichkeit_-_eine_kulturologische_Analyse_ Deutsch_-_ Italienisch_-_Polnisch (letzter Zugriff: 31.01.2020).

Deutscher, Guy (2014): Im Spiegel der Sprache. Warum die Welt in anderen Sprachen anders aussieht, 4. Aufl., München: Deutscher Taschenbuch Verlag.

Fill, Alwin Frank (2013): Linguistische Promenade - eine vergnügliche Wanderung durch die Sprachwissenschaft von Platon zu Chomsky. Berlin/ Wien: LIT Verlag.

Heringer, Hans-Jürgen u. a. (1977): Einführung in die Praktische Semantik, Heidelberg: Quelle\& Meyer.

Kuße, Holger (2012): Kulturwissenschaftliche Linguistik. Eine Einführung, Göttingen: Vandenhoeck \& Ruprecht GmbH \& Co. KG.

Maas, Utz/ Wunderlich, Dieter (1972): Pragmatik und sprachliches Handeln. Mit einer Kritik am Funkkolleg ,Sprache',Frankfurt/M: Athenäum Verlag.

Meibauer, Jörg (2001): Pragmatik, 2., verbesserte Aufl. Tübingen: Stauffenburg Verlag.

Pande, Anjali (2009): Was sagen indische Lernende, wenn sie um etwas bitten, etwas begründen oder sich entschuldigen wollen? IDV-Magazin. Beiträge der IDT 2009 Jena/Weimar - Nr. 81 Dezember 2009, S. 338-354. https://www.idvnetz.org/publikationen/magazin/IDV-Magazin81.pdf (letzter Zugriff: 31.01.2020).

Schroeder, Severin (2015): Wittgenstein: Gebrauch, Sprachspiel, Regeln. Kompa, Nikola (Hg.): Handbuch Sprachphilosophie. Stuttgart: Springer, 207-215.

Steinbach, Markus u. a. (2007): Schnittstellen der germanistischen Linguistik, Stuttgart/ Weimar: J. B. Metzler.

Zengin, Dursun (2003): Modalitätsverben im Deutschen. Atatürk Üniversitesi, Fen-Edebiyat Fakültesi Sosyal Bilimler Dergisi, Cilt: 3, Sayı: 30, 33-54.

Zengin, Dursun (2004): Präsuppositionen. A.Ü. DTCF Dergisi, Cilt: 44, Sayı: 1, 77- 93. 